nephron

Practice
Nephron 2016;134:177-182

DOI: $10.1159 / 000447757$
Received: May 4, 2016

Accepted after revision: June 20, 2016

Published online: July 26, 2016

\title{
Serum Creatinine Trajectories for Community- versus Hospital-Acquired Acute Kidney Injury
}

\author{
David G. Warnock ${ }^{a}$ T. Clark Powell ${ }^{b}$ Edward D. Siew ${ }^{f}$ John P. Donnelly ${ }^{c} d$ \\ Henry E. Wang ${ }^{\text {e Ravindra L. Mehtag }}$ \\ a Department of Medicine, ${ }^{b}$ School of Medicine, cDivision of Preventive Medicine, d Department of Epidemiology, \\ School of Public Health, and e Department of Emergency Medicine, University of Alabama at Birmingham, \\ Birmingham, Ala., ${ }^{\text {}}$ Department of Medicine, Vanderbilt University Medical Center, Nashville, Tenn., and \\ gDepartment of Medicine, University of California at San Diego, San Diego, Calif., USA
}

\section{Key Words}

Baseline serum creatinine - Serum creatinine .

Multivariable regression models $\cdot$ Creatinine trajectory

\begin{abstract}
Background: Patterns of acute kidney injury (AKI) can be distinguished by the rate of changes in the serum creation concentrations during hospitalizations. We hypothesized that the timing and values of minimum and maximum serum creatinine $(\mathrm{s} C \mathrm{r}$ ) could be used to distinguish between transient hospital-associated AKI (THA-AKI) and hospital-acquired AKI (HA-AKI). Materials and Methods: We evaluated adults admitted to 2 regionally distinct academic medical centers. Peak sCr during the hospitalization was used to define AKI, using absolute changes and timing from the minimum $\mathrm{sCr}$. $\mathrm{s} C \mathrm{r}$ trajectories were derived based on the rate of change between the minimum and peak creatinine concentrations. Results: Peak creatinine followed the minimum creatinine for HA-AKI, while the peak creatinine preceded the minimum creatinine for THA-AKI. There were 82,403 patients included in the analyses, and 53,882 (65\%) did not have AKI during the index hospitalization. There were 2,611 inpatient deaths; HA-AKI had a 4.8-fold increased risk relative to those
\end{abstract}

without AKI $(p<0.01)$, and transient AKI had a 1.6-fold increased risk for inpatient mortality relative to inpatients without AKI $(p<0.01)$. Conclusions: Patients with hospitalassociated AKI are at an increased risk for inpatient mortality. Creatinine trajectories can be used to describe the rate of development as well as recovery from inpatient AKI. The 24and 48-hour interval slopes may be early indicators of developing AKI.

(c) 2016 S. Karger AG, Basel

\section{Introduction}

Acute kidney injury (AKI) is defined by changes in serum creatinine ( $\mathrm{sCr}$ ) from a baseline value $[1,2]$. The time course of changes in $\mathrm{s} C \mathrm{r}$ concentrations has been referred to as the creatinine 'trajectory' [3-7], and is discussed in the current KDIGO AKI staging criteria [8].

Selected paper from a presentation at the 2016 AKI and CRRT UABUCSD O'Brien Center Symposium, San Diego, Calif., USA, February 16,2016 . This symposium was supported in part from a National Institutes of Health grant for the UAB-UCSD O'Brien Center for Acute Kidney Injury Research (P30 DK079337).

\section{KARGER}

(c) 2016 S. Karger AG, Basel

E-Mail karger@karger.com

www.karger.com/nef
Prof. David G. Warnock

Department of Medicine

University of Alabama at Birmingham

Room 614 ZRB, 1720 2nd Avenue South, Birmingham, AL-35294-0007 (USA)

E-Mail dwarnock@uabmc.edu 
We used datasets from 2 large academic medical centers (University of Alabama Hospital (UAH) and University of California at San Diego (UCSD)) to examine inpatient $\mathrm{sCr}$ trajectories and hypothesized that the timing and values of minimum and maximum $\mathrm{sCr}$ could be used to distinguish between transient hospital-associated AKI (THA-AKI) and hospital-acquired AKI (HA-AKI). Our objectives were to (1) describe the time course of sCr trajectories, using the temporal relationships between the minimum and peak $\mathrm{sCr}$ values, and (2) determine the associations between the patterns of AKI defined by the creatinine trajectories and inpatient mortality.

\section{Materials and Methods}

\section{Description of Cohorts and Definitions}

We examined adults admitted to UAH between October 1, 2009 and September 30, 2013, and UCSD Medical Center between January 1, 2011 and December 31, 2012, obtaining demographic information, admission and discharge dates, discharge diagnoses, procedure codes, and vital status. The Institutional Review Boards at both sites approved the use of de-identified patient information for this study.

The index admissions from UAH $(\mathrm{n}=67,019)$ and UCSD $(\mathrm{n}=$ 15,384 ) were limited to 30 days. Patients were excluded who received chronic dialysis or kidney transplantation, or had $<2$ inpatient $\mathrm{sCr}$ values, or maximum estimated glomerular filtration rates (eGFR) $<15 \mathrm{ml} / \mathrm{min} / 1.73 \mathrm{~m}^{2}$.

AKI was defined by comparing the peak sCr value to the minimum sCr for each admission [9], with the additional criteria that the elapsed time between the peak sCr and the minimum sCr was $>6 \mathrm{~h}$. We omitted patients with $\mathrm{sCr}$ values $<0.4 \mathrm{mg} / \mathrm{dl}$ and used AKI threshold criteria that reflected the minimum sCr: $0.3 \mathrm{mg} / \mathrm{dl}$ change for minimum $\mathrm{sCr}<1.0 \mathrm{mg} / \mathrm{dl}$, and $0.5 \mathrm{mg} / \mathrm{dl}$ change for minimum $\mathrm{sCr} \geq 1.0 \mathrm{mg} / \mathrm{dl}[10,11]$. Patients without significant changes in the $\mathrm{sCr}$ comprised the reference group (No-HA-AKI). $\mathrm{HA}-\mathrm{AKI}$ was defined by the minimum sCr preceding the peak $\mathrm{sCr}$, and an increased sCr above the AKI threshold. The peak preceding the minimum sCr, with a significant decrease in sCr, defined THA-AKI.

\section{Other Adjustments and Statistical Analysis}

The baseline sCr (cr-min0) for HA-AKI was defined by the timing and magnitude of the last $\mathrm{sCr}$ that still remained within the AKI threshold following the minimum sCr. The average minimum sCr for HA-AKI $(0.96 \mathrm{mg} / \mathrm{dl})$ was observed $43 \mathrm{~h}$ following admission, and the cr-min0 $(1.02 \mathrm{mg} / \mathrm{dl})$ averaged $62 \mathrm{~h}$ after admission. Maximal sCr values were calculated at 24 -hour intervals after $\mathrm{cr}-\mathrm{min} 0$ for describing the sCr trajectory. Similarly, minimal sCr values were calculated at 24-hour intervals after the peak $\mathrm{sCr}$ values for describing recovery from the peak sCr.

We compared baseline characteristics of patients using chisquare tests and analysis of variance. The Dunnett test was used for multiple comparisons, with minimal statistical significance set at $\mathrm{p} \leq 0.05$. Mean values are presented as $\pm 1 \mathrm{SD}$, and medians with 25th and 75th-centiles. Incident rates and incident rate ratios were obtained with Poisson regression models and presented with $95 \%$ CIs. Statistical analyses were performed with Stata version 14.1 (StataCorp, College Station, Tex., USA).

\section{Results}

The baseline characteristics are shown in table 1. Patients with AKI were older, more likely to be male, longer lengths of stay, and to receive intensive than the reference group. The proportion with maximum eGFR $<60 \mathrm{ml} /$ $\mathrm{min} / 1.73 \mathrm{~m}^{2}$ was similar for THA-AKI and HA-AKI, and greater than the reference group. HA-AKI was associated with $13 \%$, THA-AKI with $4.0 \%$ and No-HA-AKI with $1.3 \%$ inpatient mortality rates.

Figure 1 illustrates the $\mathrm{sCr}$ trajectories for HA-AKI $(8,465$ patients; closed symbols) and THA-AKI (20,056 patients; open symbols). Also shown is the time course for No-HA-AKI (53,882 patients; gray symbols), with a shorter length of stay than either THA-AKI or HA-AKI. The time-course is distinct for THA-AKI compared to HA-AKI, with marked separation between the timing of the minimum and maximum sCr values.

Figure 2 shows the $\mathrm{Cr}$ trajectory for HA-AKI, with the minimum, peak and last $\mathrm{sCr}$ values. The $\mathrm{cr}-\mathrm{min} 0$ was the baseline sCr used to define the sCr trajectory. On average, sCr increased by $0.89 \pm 1.02 \mathrm{mg} / \mathrm{dl}$ over $95 \pm 81 \mathrm{~h}$ from the baseline sCr for HA-AKI. Also shown are the highest sCr values recorded during the indicated 24 -hour intervals following the cr-min0. This slope of the sCr trajectory was steeper for the first 24- or 48-hour interval than that calculated using the peak sCr.

Recovery from HA-AKI compared the last $\mathrm{sCr}$ and the peak sCr values, and was $0.33 \pm 0.59 \mathrm{mg} / \mathrm{dl}$ over $28 \pm 29 \mathrm{~h}$ following the peak sCr value.

The minimum, peak and last $\mathrm{sCr}$ are shown along with the minimal $\mathrm{sCr}$ values in each of the indicated time intervals for THA-AKI in figure 3. On average, $\mathrm{sCr}$ decreased by $0.89 \pm 1.21 \mathrm{mg} / \mathrm{dl}$ to the minimum sCr at $95 \pm$ $81 \mathrm{~h}$ following the peak sCr. The initial rate of recovery was more rapid and then it attenuated over subsequent days.

\section{Discussion}

We have previously described patterns of inpatient AKI [9], and now extend these descriptions to index admissions for another academic medical center. De- 
Table 1. Characteristics and mortality rates for inpatients without, or with AKI (82,403 patients; 2,611 inpatient deaths (3.2\%))

\begin{tabular}{|c|c|c|c|}
\hline \multicolumn{4}{|c|}{ Demographic characteristics, time course and changes in sCr } \\
\hline Age, years ${ }^{b}$ & $56(42-68)$ & $58(44-69)$ & $59(48-70)^{\mathrm{c}}$ \\
\hline Male sex, n (\%) & $27,287(51)$ & $12,077(60)$ & $4,521(53)^{c}$ \\
\hline Whites, other patients, n (\%) & $37,574(70)$ & $13,564(67)$ & $5,690(67)$ \\
\hline Length of stay, days & $3.1(2.0-5.1)$ & $6.2(4.0-11.0)$ & $7.1(4.1-12.0)^{\mathrm{c}}$ \\
\hline Intensive care unit stay, $\mathrm{n}(\%)$ & $9,278(17)$ & $7,969(39)$ & $3,691(44)^{c}$ \\
\hline Max-eGFR, $\mathrm{ml} / \mathrm{min} / 1.73 \mathrm{~m}^{2}$ & $99(80-116)$ & $98(73-117)$ & $93(69-111)^{c}$ \\
\hline Max-eGFR <60 ml/min/1.73 $\mathrm{m}^{2}(60), \mathrm{n}(\%)$ & $5,998(11)$ & $3,529(17)$ & $1,666(20)^{\mathrm{c}}$ \\
\hline \multicolumn{4}{|l|}{ Inpatient mortality, 30 day length of stay } \\
\hline Inpatient deaths, n (\%) & $673(1.3)$ & $817(4.0)$ & $1,121(13)^{\mathrm{C}}$ \\
\hline
\end{tabular}

Values are given as number (percentage), means $\pm 1 \mathrm{SD}$, medians with (25th-75th centile), or means $(95 \% \mathrm{CI})$ for incident rates and incident rate ratios.

Max-eGFR = Maximum eGFR for admission, based on minimum sCr for the admission.

${ }^{a}$ Row percentages; all others are column percentages.

${ }^{b} \mathrm{p}<0.001$ for comparisons of characteristics between each group by analysis of variance for means, signed rank test for me- dians, and chi-square test for proportions; shown as bold text, relative to No-AKI which served as the reference group.

${ }^{c} \mathrm{p}<0.05$ for comparison between THA-AKI and HA-AKI (Duncan test for multiple comparisons) or chi-square test of proportions.

${ }^{d}$ Race or ethnic group was self-reported, and further classified as black or non-black. spite differences in demographics and case mix, the AKI patterns and $\mathrm{sCr}$ trajectories were similar at both sites.

The definition of AKI is based on changes in $\mathrm{sCr}$ from a reference value [8]. The magnitude and timing of the peak and sCr with respect to the reference point are needed for characterizing sCr trajectories and AKI patterns: $\mathrm{sCr}$ is a vector with both magnitude $(\mathrm{mg} / \mathrm{dl})$ and time dimension, defined as the elapsed time after admission. Ambulatory $\mathrm{sCr}$ values obtained before admission have been used as a baseline $\mathrm{sCr}$ for characterizing AKI in the hospital setting $[1,2,12]$, but these scalars lack the relevant time dimension required to define $\mathrm{sCr}$ trajectories. Creatinine kinetics and trajectories have been explicitly considered in some analyses of AKI $[5,7,10,13,14]$, and addressed in the KDIGO AKI criteria [8], but most studies have not considered sCr trajectories, and have only used the scalar differences between maximum and baseline $\mathrm{sCr}$.

We do not equate the minimum sCr value for a given admission to the 'baseline' $\mathrm{SCr}$ value. While the minimum sCr has evident utility for separating THA-AKI from HAAKI, it may not be the optimal reference point for defin-

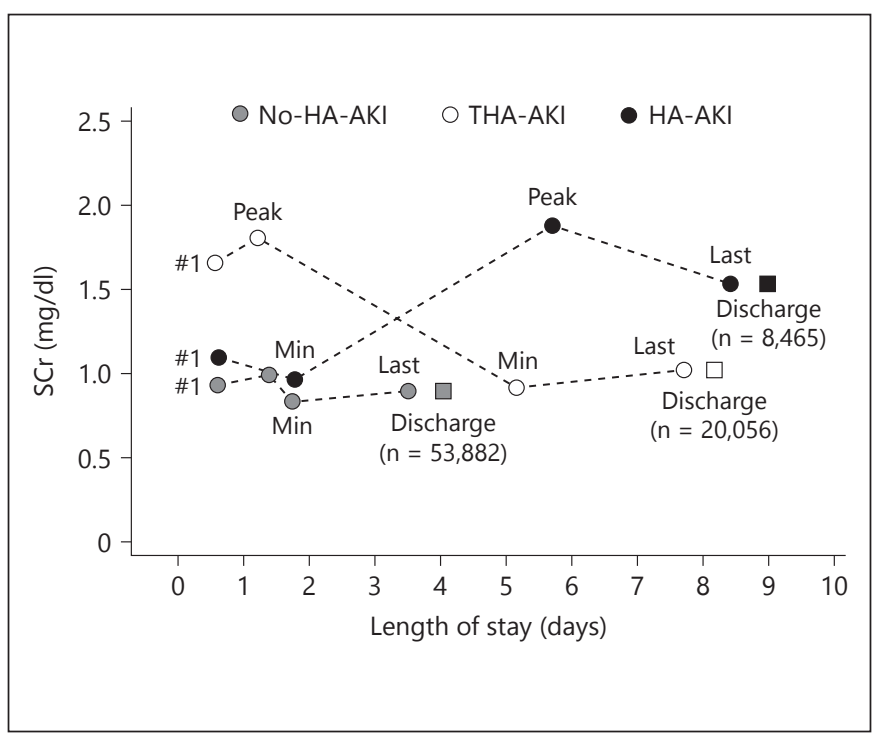

Fig. 1. Creatinine trajectories by AKI types, combined cohort (82,402 patients). AKI patterns were defined by the temporal sequence of the minimum (Min) and maximum (Peak) inpatient $\mathrm{sCr}$ values. Also shown are the first (\#1) and last $\mathrm{sCr}$ values. Squares indicate the discharge date. 


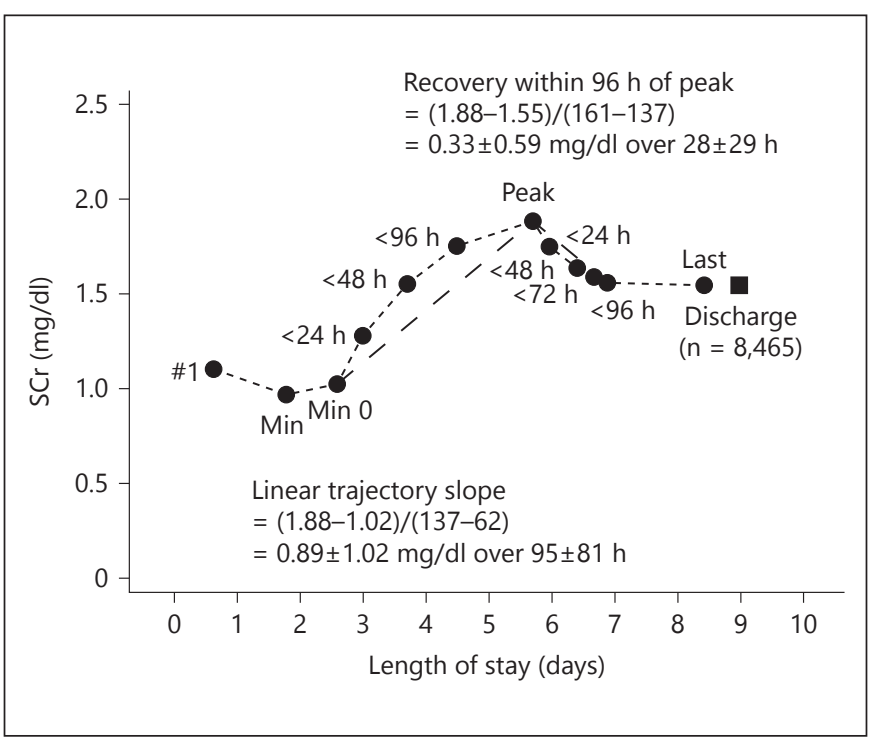

Fig. 2. Creatinine trajectory for HA-AKI (8,453 patients). HA-AKI was defined by the minimum (Min) $\mathrm{sCr}$ preceding the maximum (Peak) sCr during the admission. Also shown are the first (\#1) and last sCr values, and the discharge date. The intervening time points represent the minimum $\mathrm{sCr}$ values during the indicated intervals in hours after the peak value, and the maximum sCr values during the indicated intervals in hours after the minimum value. The dashed upward diagonal line is an estimate of the slope of the creatinine trajectory from min 0 value to peak value $(0.89 \mathrm{mg} / \mathrm{dl} / 95$ $\mathrm{h})$. The dashed downward diagonal line is an estimate of the recovery from peak $\mathrm{sCr}(0.33 \mathrm{mg} / \mathrm{dl}$ over $28 \mathrm{~h})$.

ing sCr trajectories, assessing recovery from $\mathrm{AKI}$, or staging of the severity of HA-AKI. For example, the delay after the minimum sCr before the HA-AKI threshold is reached suggests that the timing and magnitude of the last $\mathrm{sCr}$ before a significant increase in $\mathrm{sCr}$, which we term cr-min0 in figure 2, constitute a better baseline sCr than the minimum sCr for HA-AKI. Further work is needed to define the baseline or reference $\mathrm{sCr}$ values especially for the staging of HA-AKI, but this approach may be helpful in defining a baseline sCr for patients who lack pre-admission data. The creatinine trajectory (heavy dashed line) for HA-AKI is curvilinear (fig. 2), and may not be accurately represented by the simple rise and run of sCr between the minimum and peak sCr values, which can be considered to be a minimal estimation of the slope of the creatinine trajectory.

A notable strength of the current analysis is the use of sCr trajectories, rather than discharge codes to define AKI $[15,16]$. Nevertheless, our study has several limitations. We could not determine the etiology of AKI, urine output, fluid administration or other medications from

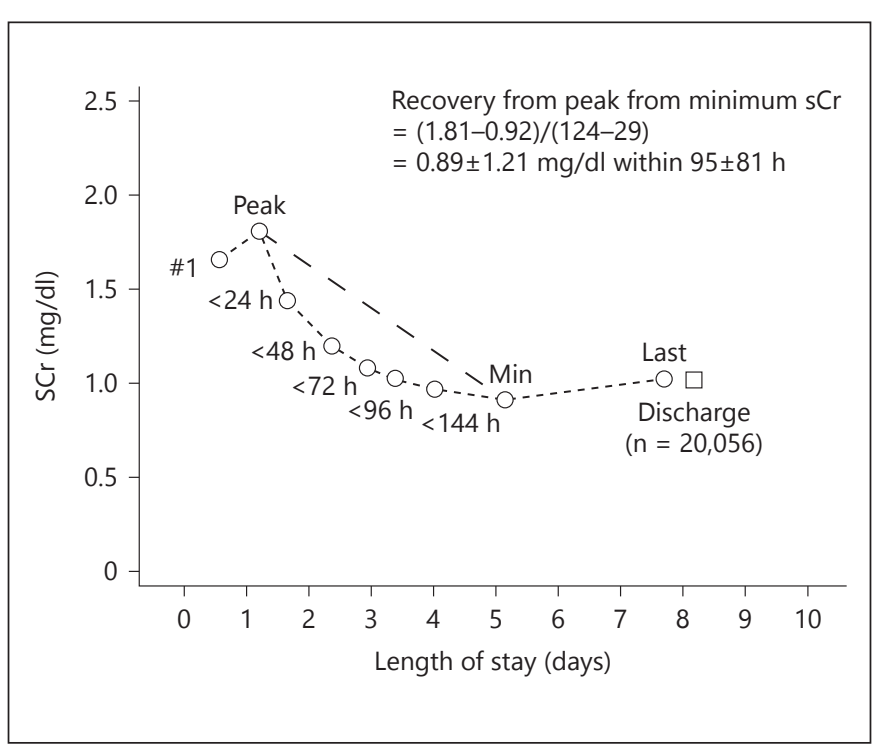

Fig. 3. Creatinine trajectory for THA-AKI (20,255 patients). THAAKI was defined by the maximum (Peak) sCr preceding the minimum (Min) sCr during the admission. Also shown are the first (\#1) and last sCr values, and the discharge date. The intervening time points represent the maximum $\mathrm{sCr}$ values during the indicated intervals (hours) following the peak sCr. The dashed downward diagonal line is an estimate of the recovery from peak to minimum $\mathrm{sCr}(0.89 \mathrm{mg} / \mathrm{dl}$ within $95 \mathrm{~h})$.

the current inpatient datasets. The frequency and timing of inpatient sCr determinations are dependent on local clinical practices, which could be a source of residual confounding $[17,18]$. Follow-up status after discharge and pre-hospitalization $\mathrm{sCr}$ values are not currently available.

Changes in sCr depend on factors that modify creatinine generation (body composition, muscle wasting, and acute muscle injury) [19]. Changes in the volume of distribution of creatinine (dehydration or volume expansion) may occur, especially in the elderly [20] and critically ill patients with prolonged hospitalizations [21-23], which could lead to false-negative ascertainment. To address these limitations, we have excluded patients with minimum sCr values $<0.4 \mathrm{mg} / \mathrm{dl}$, or with a maximal eGFR (based on the minimum $\mathrm{sCr}$ ) $<15 \mathrm{ml} / \mathrm{min} / 1.73 \mathrm{~m}^{2}$ throughout the duration of hospitalization. In addition, we limited the length of stay to 30 days, and acknowledge that outcome misclassification is inherent in the use of vital status administrative coding at the time of discharge or other deposition [24]. 
In conclusion, we have described the patterns of AKI based on their creatinine trajectories, and have illustrated the value of defining patterns of AKI based on the timing and magnitude of changes in $\mathrm{sCr}$ values for each subject during a single admission. Patients with hospital-associated AKI are at increased risk for inpatient mortality. Creatinine trajectories can be used to describe the rate of development as well as recovery from inpatient AKI. The 24- and 48-hour interval slopes may be early indicators of developing AKI. Further work is needed, but the use of creatinine trajectories may support the development of real-time, dynamic tools for assessing the risks associated with evolving AKI, which is a pressing need for current AKI research [25-27].

\section{Acknowledgments}

This study was presented at the AKI as a Quality Paradigm: Round Table Conference at the 21st International Conference on Continuous Renal Replacement Therapies San Diego, Calif., USA, February 15-16, 2016. We thank Darlene Green and Stephen Duncan for their assistance with the data set.

\section{Authorship}

All authors participated in the study design, analysis and writing of the manuscript. No other persons participated in the analyses, drafting or revision of the manuscript.

\section{Support}

A Kidney Research Student Scholar Grant from the American Society of Nephrology Foundation supported T.C. Powell during a research elective. J.P. Donnelly received support from the Agency for Healthcare Research and Quality, Rockville, Maryland (T32HS013852). The Veterans Affairs HSR\&D IIR 13-073, and the Vanderbilt Center for Kidney Disease and Integrated Program for AKI Research supported Dr. E.D. Siew. Dr. H.E. Wang received grant support from the National Institute for Nursing Research (R01-NR012726). Dr. D.G. Warnock is a Professor of Medicine (Emeritus). We also acknowledge support from the UAB/UCSD O’Brien Center Kidney Research (P30 DK079337).

\section{Disclosure Statement}

None of the authors disclose any conflicts of interest relevant to this report.

\section{References}

$\checkmark 1$ Siew ED, Matheny ME, Ikizler TA, Lewis JB, Miller RA, Waitman LR, Go AS, Parikh CR, Peterson JF: Commonly used surrogates for baseline renal function affect the classification and prognosis of acute kidney injury. Kidney Int 2010;77: 536-542.

2 Siew ED, Matheny ME: Choice of reference serum creatinine in defining acute kidney injury. Nephron 2015;131:107-112.

-3 Guitterez NV, Diaz A, Timmis GC, O’Neill WW, Stevens MA, Sandberg KR, McCullough PA: Determinants of serum creatinine trajectory in acute contrast nephropathy. J Interv Cardiol 2002;15:349-354.

$\checkmark 4$ Li L, Astor BC, Lewis J, Hu B, Appel LJ, Lipkowitz MS, Toto RD, Wang X, Wright JT, Jr, Greene TH: Longitudinal progression trajectory of GFR among patients with CKD. Am J Kidney Dis 2012;59:504-512.

$\checkmark 5$ Bojan M, Lopez-Lopez V, Pouard P, Falissard B, Journois D: Limitations of early serum creatinine variations for the assessment of kidney injury in neonates and infants with cardiac surgery. PLoS One 2013; 8:e79308.

6 Onuigbo MA, Agbasi N: Diabetic nephropathy and CKD-analysis of individual patient serum creatinine trajectories: a forgotten diagnostic methodology for diabetic CKD prognostication and prediction. J Clin Med 2015; 4:1348-1368.
7 Endre ZH, Pianta TJ, Pickering JW: Timely diagnosis of acute kidney injury using kinetic eGFR and the creatinine excretion to production ratio, E/eG - creatinine can be useful! Nephron 2016;132:312-316.

\$8 KDIGO Work Group: KDIGO clinical practice guideline for acute kidney injury. Kidney Int Suppl 2012;2:1-138.

-9 Warnock DG, Powell TC, Donnelly JP, Wang HE: Categories of hospital-associated acute kidney injury: time course of changes in serum creatinine values. Nephron 2015;131: 227-236.

10 Zeng X, McMahon GM, Brunelli SM, Bates DW, Waikar SS: Incidence, outcomes, and comparisons across definitions of AKI in hospitalized individuals. Clin J Am Soc Nephrol 2014;9:12-20.

11 Lin J, Fernandez H, Shashaty MG, Negoianu D, Testani JM, Berns JS, Parikh CR, Wilson FP: False-positive rate of AKI using consensus creatinine-based criteria. Clin J Am Soc Nephrol 2015;10:1723-1731.

12 Ricci Z, Cruz DN, Ronco C: Classification and staging of acute kidney injury: beyond the RIFLE and AKIN criteria. Nat Rev Nephrol 2011;7:201-208.

13 Waikar SS, Bonventre JV: Creatinine kinetics and the definition of acute kidney injury. J Am Soc Nephrol 2009;20:672-679.

14 Chen S: Retooling the creatinine clearance equation to estimate kinetic GFR when the plasma creatinine is changing acutely. J Am Soc Nephrol 2013;24:877-888.

-15 Koulouridis I, Price LL, Madias NE, Jaber BL: Hospital-acquired acute kidney injury and hospital readmission: a cohort study. Am J Kidney Dis 2015;65:275-282.

16 Waikar SS, Wald R, Chertow GM, Curhan GC, Winkelmayer WC, Liangos O, Sosa MA, Jaber BL: Validity of international classification of diseases, ninth revision, clinical modification codes for acute renal failure. J Am Soc Nephrol 2006;17:1688-1694.

17 Chertow GM, Burdick E, Honour M, Bonventre JV, Bates DW: Acute kidney injury, mortality, length of stay, and costs in hospitalized patients. J Am Soc Nephrol 2005;16:3365-3370.

- 18 Coca SG, Peixoto AJ, Garg AX, Krumholz HM, Parikh CR: The prognostic importance of a small acute decrement in kidney function in hospitalized patients: a systematic review and meta-analysis. Am J Kidney Dis 2007;50: 712-720.

19 Star RA: Treatment of acute renal failure. Kidney Int 1998;54:1817-1831.

20 Chao CT, Wu VC, Lai CF, Shiao CC, Huang TM, Wu PC, Tsai IJ, Hou CC, Wang WJ, Tsai HB, Lin YF, Chiang WC, Lin SL, Tsai PR, Ko WJ, Wu MS, Wu KD; NSARF Group: Advanced age affects the outcomepredictive power of RIFLE classification in geriatric patients with acute kidney injury. Kidney Int 2012;82:920-927. 
-21 Doi K, Yuen PS, Eisner C, Hu X, Leelahavanichkul A, Schnermann J, Star RA: Reduced production of creatinine limits its use as marker of kidney injury in sepsis. J Am Soc Nephrol 2009;20:1217-1221.

22 Macedo E, Bouchard J, Soroko SH, Chertow GM, Himmelfarb J, Ikizler TA, Paganini EP, Mehta RL; Program to Improve Care in Acute Renal Disease Study: Fluid accumulation, recognition and staging of acute kidney injury in critically-ill patients. Crit Care 2010;14:R82.
23 Prowle JR, Kolic I, Purdell-Lewis J, Taylor R, Pearse RM, Kirwan CJ: Serum creatinine changes associated with critical illness and detection of persistent renal dysfunction after AKI. Clin J Am Soc Nephrol 2014;9:10151023.

24 Waikar SS, Liu KD, Chertow GM: The incidence and prognostic significance of acute kidney injury. Curr Opin Nephrol Hyperten 2007; 16:227-236
25 Chertow GM, Winkelmayer WC: Early to dialyze: healthy and wise? JAMA 2016;315: 2171-2172.

26 Mehta RL: Renal-replacement therapy in the critically ill - does timing matter? N Engl J Med 2016, Epub ahead of print.

27 Warnock DG: The pressing need for real-time risk assessment of hospital-acquired acute kidney injury. Nephrol Dial Transplant 2016, in press. 